\title{
Carbonic anhydrase IX-directed immunoliposomes for targeted drug delivery to human lung cancer cells in vitro
}

This article was published in the following Dove Press journal:

Drug Design, Development and Therapy

22 July 2014

Number of times this article has been viewed

\author{
Blenda Chi Kwan Wong' \\ Hongqi Zhang' \\ Ling Qin² \\ Hubiao Chen' \\ Chen Fang' \\ Aiping Lu' \\ Zhijun Yang' \\ 'School of Chinese Medicine, \\ Hong Kong Baptist University, \\ Kowloon Tong, Kowloon, Hong Kong; \\ ${ }^{2}$ Department of Orthopaedics and \\ Traumatology, The Chinese University \\ of Hong Kong, Prince of Wales \\ Hospital, Shatin, New Territories, \\ Hong Kong
}

\begin{abstract}
Targeted drug delivery to cancer cells by use of antibody-conjugated liposomes (immunoliposomes) has attracted considerable interest in recent years. Despite increasing efforts in developing immunoliposomes as drug carriers, the investigation of useful tumor-associated antigen targets is far from complete. Carbonic anhydrase IX (CA IX) is a cell surface antigen characterized by hypoxia-induced expression in many solid tumors. This study investigated the feasibility of CA IX-directed immunoliposomes for targeted delivery of docetaxel to human lung cancer cells in vitro. Docetaxel-loaded immunoliposomes targeting CA IX were developed with an encapsulation efficiency of $84.4 \pm 3.9 \%$ and an average particle size of $143.9 \pm 11.1 \mathrm{~nm}$. Using fluorescence-based flow cytometry, the in vitro binding activity of the immunoliposomes was found to be significantly higher (by 1.65-fold) than that of the nontargeted liposomes in CA IX-positive lung cancer cells, whereas no such difference was observed between the two groups when CA IX was not expressed. Furthermore, immunoliposomal docetaxel exhibited the strongest growth inhibitory effect against CA IX-positive lung cancer cells when compared with nontargeted liposomal docetaxel or free docetaxel solution. These data suggested that CA IX-directed immunoliposomes could serve as a promising drug delivery system for targeted killing of lung cancer cells.
\end{abstract}

Keywords: cancer chemotherapy, conjugation, liposome, nanotechnology, cell surface antigen, hypoxia

\section{Introduction}

Carbonic anhydrase IX (CA IX) as a cell surface antigen protein has been extensively studied in relation to its expression in different tumor types, its correlation with tumorrelated hypoxia and prognostic factors, and its potential application as a therapeutic target. The biological properties of CA IX have made it a favorable target for cancer therapy. As a transmembrane protein, CA IX is easily accessible by antibodies or small molecule inhibitors. The N-terminal region of CA IX containing the catalytic domain is exposed to the extracellular side, contributing to its role in tumor-associated pericellular acidification by the conversion of carbonic dioxide to bicarbonate and proton. ${ }^{1}$ CA IX is expressed ectopically in various commonly occurring carcinomas, including those of the kidney, ${ }^{2}$ colon, ${ }^{3}$ breast,${ }^{4}$ and lung, ${ }^{5}$ and it is often correlated with poor prognosis in cancer patients. ${ }^{6-10}$ In particular, CA IX as a marker of tumor hypoxia was previously observed in $80 \%$ of non-small-cell lung carcinomas (NSCLC). ${ }^{5,11,12}$ In contrast, in normal tissue, moderate to high expression of CA IX is restricted to the basolateral surface of gastric, intestinal, and gallbladder epithelia; ${ }^{13}$ diffuse and weak expression of CA IX is observed only in the epithelia of pancreatic ducts and male reproductive organs. ${ }^{14,15}$ In view of these findings, CA IX-mediated targeted therapy represents a promising strategy for cancer treatment. 
In fact, immunotherapy using CA IX-specific monoclonal antibodies can be exploited through different mechanisms. Direct binding of antibodies to CA IX can elicit an antitumor response due to antibody-dependent cell-mediated cytotoxicity. ${ }^{16}$ Such a tool has been examined in Phase I and II trials, which showed that use of this antibody as an adjuvant therapy could increase patient survival for metastatic renal cell carcinoma. ${ }^{17}$ Alternatively, antibodies targeting the catalytic domain of CA IX can disrupt its tumorigenic functions, compromising the survival of hypoxic tumor cells. ${ }^{18}$ Furthermore, the ability of antibodies to undergo receptor-mediated internalization allows the delivery of cytotoxic drugs to cancer cells. This targeting potential can be utilized to increase drug accumulation in the tumor while reducing unwanted nonspecific toxicity in normal tissue.

One of the most commonly used drug carriers for targeted cancer therapy is liposomes, which offer significant advantages such as good biocompatibility, low toxicity, and low immunogenicity. ${ }^{19}$ Particularly, the generation of immunoliposomes by coupling antibodies to the liposomal surface has opened up a new venue for the concept of cancer cell targeting. ${ }^{20}$ Shinkai et al previously developed CA IXdirected immunoliposomes for targeted delivery of submicron magnetic particles to renal cell carcinoma tissue as a form of magnetic field-induced hyperthermia therapy and demonstrated their feasibility in the suppression of tumor tissue and extension of survival time in animal models. ${ }^{21}$ Since then, however, there have been no publications documenting the use of CA IX for immunoliposome-based therapy in other types of cancer.

In the present study, we hypothesized that CA IXdirected immunoliposomes could facilitate drug uptake in human NSCLC cells. For in vitro experiments, human NSCLC cells can be treated with hypoxia to induce the expression of CA IX. ${ }^{22}$ Docetaxel (DTX) is a conventional anticancer drug used for the treatment of NSCLC. The CA IX-directed immunoliposomes encapsulating DTX were developed using the ethanol injection method ${ }^{23}$ combined with the postinsertion technique. ${ }^{24}$ The immunoliposomes were characterized with respect to their particle size and drug encapsulation efficiency. Fluorescence-based flow cytometry assay was carried out to compare the cell binding affinity of the immunoliposomes with that of the nontargeted liposomes in NSCLC cells with and without the expression of CA IX. The drug uptake mediated by immunoliposomes against CA IX in NSCLC cells was evaluated using the cytotoxicity assay.

\section{Materials and methods Materials}

Soybean phosphatidylcholine (SPC) was purchased from Taiwei Pharmaceutical Co, Ltd (Shanghai, People's Republic of China). N-[(3-maleimide-1-oxopropyl)aminopropyl polyethyleneglycol-carbamyl] distearoylphosphatidylethanolamine (DSPE-PEG-MAL) was purchased from NOF America Corporation (White Plains, NY, USA). DTX was supplied by LC Laboratories (Woburn, MA, USA). The rabbit monoclonal anti-CA IX antibody targeting the $\mathrm{N}$-terminal region of the antigen protein that is exposed to the extracellular side was obtained from Abcam (Cambridge, UK). The goat antirabbit horseradish peroxidase (HRP)-conjugated secondary antibody was obtained from Bio-rad Laboratories (Hercules, CA, USA). Dithiotreitol (DTT) was purchased from Sigma-Aldrich (St Louis, MO, USA). The lipophilic near-infrared fluorescent dye $1,1^{\prime}$-dioctadecyltetramethyl indotricarbocyanine iodide (DiR) used for labeling liposomes was supplied by Caliper LifeSciences (Hopkinton, MA, USA). Acetonitrile of HPLC grade and ethyl acetate were obtained from Anaqua Chemicals Supply (Houston, TX, USA). Dimethyl sulfoxide (DMSO) was purchased from Sigma-Aldrich. Ultrapure water was generated by a Millipore water purification system (EMD Millipore, Billerica, MA, USA).

\section{Preparation of liposomes}

DTX-encapsulated liposomes were prepared by the ethanol injection method..$^{23}$ Briefly, $200 \mathrm{mg}$ of SPC and $16 \mathrm{mg}$ of DTX (at a lipid-to-drug weight ratio of 12.5:1) were dissolved in $0.5 \mathrm{~mL}$ of ethanol. The mixture was rapidly injected into a $10 \mathrm{~mL}$ magnetically stirred phosphate buffered saline solution (PBS, pH 7.1) using a syringe needle. Liposome suspension was easily identified by the appearance of the characteristic opalescence of a colloidal dispersion. After stirring for an hour, the liposome suspension was passed through a $0.2 \mu \mathrm{m}$ pore size filter once and then $0.1 \mu \mathrm{m}$ pore size filters (Whatman, Maidstone, Kent, UK) five times sequentially under nitrogen gas using an extruder (Northern Lipids Inc., Burnaby, BC, Canada) to generate unilamellar vesicles of low polydispersity. Unentrapped DTX was removed from the liposome suspension by Sephadex G-25 gel filtration chromatography (PD-10 Desalting Column; GE Healthcare, Little Chalfont, Buckinghamshire, UK). DiR-labeled liposomes were prepared as above, with the fluorescent dye being dissolved in PBS at a concentration of $0.4 \mathrm{mg} / \mathrm{mL}$. 


\section{Incorporation of antibody-conjugated micelles into preformed liposomes}

Immunoliposomes were generated using the postinsertion technique. ${ }^{24}$ To form DSPE-PEG-MAL micelles, a dried lipid film containing DSPE-PEG-MAL was hydrated in PBS ( $\mathrm{pH} 6.6,0.01 \mathrm{M}$ EDTA) at a concentration of $6 \mathrm{mM}$ (above the critical micellar concentration) with heating at $65^{\circ} \mathrm{C}$ for half an hour. Conjugation of the anti-CA IX antibody to DSPE-PEG-MAL in the preformed micelles was carried out using the sulfhydryl reactive chemistry. ${ }^{25}$ The antibody was cleaved at the hinge region by reacting with the reducing agent DTT $(80 \mathrm{mM})$ at room temperature for 2 hours. Unreacted DTT was then removed from reduced antibody by ethyl acetate extraction. Briefly, one volume of reduced antibody was mixed with four volumes of ethyl acetate, followed by centrifugation at 9,279 $\mathrm{g}$ for 2 minutes to achieve phase separation and removal of the organic phase. This extraction was repeated four more times to ensure the complete removal of unreacted DTT. The reduced antibody was then conjugated to DSPE-PEG-MAL micelles at a molar ratio of $1: 100$ by incubation at room temperature with gentle agitation for 24 hours. To prepare CA IX-directed immunoliposomes (targeted liposomes), antibody-conjugated micelles were incubated with preformed liposomes at a SPC:DSPE-PEGMAL molar ratio of $31: 1$ at $60^{\circ} \mathrm{C}$ for 2 hours. In parallel, nontargeted liposomes were obtained by incubating unmodified micelles with preformed liposomes at the same molar ratio under the same conditions. Free antibody or micelles were then removed by gel filtration on a Sepharose CL-4B column (Sepharose CL-4B, Sigma-Aldrich; Empty PD-10 Column, GE Healthcare). The collected fractions were analyzed for DTX by ultra-performance liquid chromatography (UPLC) and for antibody by Western blot analysis as described below.

The antibody-liposome coupling efficiency was evaluated using Western blot analysis. The immunoliposome samples with and without Sepharose CL-4B gel filtration were loaded into $10 \%$ sodium dodecyl sulfate polyacrylamide gel electrophoresis (SDS-PAGE) gels under reducing conditions and then electrotransferred to polyvinylidene difluoride (PVDF) membranes (Amersham Biosciences, GE Healthcare). The blots were blocked with 5\% nonfat dry milk for 30 minutes, followed by 1 hour room temperature incubation with the goat antirabbit HRP-conjugated secondary antibody. SuperSignal West Pico Chemiluminescent Substrate (Thermo Fisher Scientific, Waltham, MA, USA) was used for HRP-based detection of the anti-CA IX antibody that had been coupled to liposomes. The coupling efficiency was assessed by comparing the eluted immunoliposome sample against the unfiltered immunoliposome sample at the same DTX amount and using serial dilutions of the unfiltered immunoliposome sample as antibody standards.

\section{Characterization of liposomes}

Targeted and nontargeted DTX-loaded liposomes were assessed for particle size distribution using the Delsa Nano HC Particle Analyzer (Beckman Coulter, Brea, CA, USA). Drug encapsulation efficiency (EE) was determined by the Sephadex G-25 gel filtration method (PD-10 Desalting Column, GE Healthcare) based upon the following formula:

$$
\begin{aligned}
\operatorname{EE~}(\%)= & (\text { amount of entrapped drug }) / \\
& (\text { amount of total drug used }) \times 100 \\
= & \mathrm{C}_{\mathrm{e}} \mathrm{V}_{\mathrm{e}} / \mathrm{C}_{\mathrm{t}} \mathrm{V}_{\mathrm{t}} \times 100
\end{aligned}
$$

where $\mathrm{C}_{\mathrm{e}}$ and $\mathrm{V}_{\mathrm{e}}$ represent the DTX concentration and volume of the eluted liposome suspension after gel filtration, respectively, and $\mathrm{C}_{\mathrm{t}}$ and $\mathrm{V}_{\mathrm{t}}$ represent the DTX concentration and volume of the original liposome suspension before extrusion, respectively. For DTX quantification, the liposome samples were diluted tenfold with acetonitrile to release entrapped DTX, followed by UPLC (ACQUITY UPLC System, Waters, Milford, MA, USA) using the ACQUITY UPLC BEH Shield RP18 column $(1.7 \mu \mathrm{m}$, $2.1 \mathrm{~mm} \times 100 \mathrm{~mm}$, Waters) with UV detection at $227 \mathrm{~nm}$. The injection volume was $2 \mu \mathrm{L}$, and the mobile phase consisted of water and acetonitrile containing $0.1 \%$ formic acid in a ratio of 50:50 (v:v [volume:volume]) at a flow rate of $0.5 \mathrm{~mL} / \mathrm{minute}$.

\section{Detection of CA IX expression in cell cultures}

The human NSCLC cell line A549 was kindly provided by the Department of Anatomical and Cellular Pathology, The Chinese University of Hong Kong. The cells were grown in DMEM with GlutaMAX supplemented with $10 \%$ fetal bovine serum, $100 \mathrm{U} / \mathrm{mL}$ penicillin, and $100 \mu \mathrm{g} / \mathrm{mL}$ streptomycin (Invitrogen, Life Technologies, Carlsbad, CA, USA). Since A549 cells normally do not express CA IX under normoxic conditions (humidified air with $5 \% \mathrm{CO}_{2}$ ), cell cultures were incubated under hypoxic conditions for induction of CA IX using a Modular Incubator Chamber (MIC-101; BillupsRothenberg Inc., Del Mar, CA, USA) purged with $1 \% \mathrm{O}_{2}$, $5 \% \mathrm{CO}_{2}$, and balance $\mathrm{N}_{2}$. Parallel exposures to normoxia and hypoxia were performed at $37^{\circ} \mathrm{C}$ for 20 hours, followed by CA IX detection using Western blot analysis. Cells were 
lysed with RIPA buffer (Thermo Fisher Scientific) and protein concentrations were determined by the bicinchoninic acid method (Pierce BCA Protein Assay Kit; Thermo Fisher Scientific). The protein samples $(50 \mu \mathrm{g}$ each) were separated on $10 \%$ SDS-PAGE gels under reducing conditions and then electrotransferred to PVDF membranes. After blocking in a solution of $5 \%$ nonfat dry milk, the blots were incubated with the rabbit monoclonal anti-CA IX antibody at $4^{\circ} \mathrm{C}$ overnight. After washing, the blots were incubated with the goat antirabbit HRP-conjugated secondary antibody at room temperature for 1 hour. Chemiluminescence detection was performed using the SuperSignal West Pico Chemiluminescent Substrate.

\section{Cell binding assay}

Comparison of the binding affinities of targeted and nontargeted DTX liposomes to CA IX-positive and CA IX-negative A549 cells was performed using the fluorescence-based flow cytometry method. A549 cells were seeded at a density of $2.5 \times 10^{5}$ cells per well in six-well culture plates and allowed to grow overnight. CA IX-positive and CA IX-negative cells were generated by parallel treatments with hypoxia and normoxia, respectively, as described above. As CA IX antigen has been found to exist in both cell-associated and cell-free forms, ${ }^{26}$ the cells were then washed with PBS to remove the cell-free form that could otherwise interfere with cell binding. Exposure to DiR-labeled liposomes $(5 \mu \mathrm{M})$, targeted or nontargeted, was then performed in triplicate at $4{ }^{\circ} \mathrm{C}$ for 2 hours. Cells treated with PBS only were included as negative controls. After removal of the unbound liposomes using PBS, the cells were harvested for flow cytometry analysis (BD FACSCanto Flow Cytometer; BD Biosciences, San Jose, CA, USA). Fluorescent signals were acquired by collecting 10,000 events per sample.

\section{Cytotoxicity study}

The cytotoxic effects of targeted liposomal DTX, nontargeted liposomal DTX, and free DTX on CA IXpositive and CA IX-negative A549 cells were determined using the colorimetric 3-(4,5-dimethylthiazol-2-yl)-2,5 -diphenyltetrazolium bromide (MTT, Invitrogen) assay. A549 cells were seeded at a density of $4 \times 10^{3}$ cells per well in 96-well culture plates and allowed to grow overnight. Parallel incubations in hypoxia and normoxia were then performed to obtain CA IX-positive and CA IX-negative cells, respectively. The cells were washed with PBS to remove cell-free CA IX, followed by 2 hours incubation with targeted DTX liposomes, nontargeted DTX liposomes, or free DTX solution at different concentrations in triplicate cultures at $4^{\circ} \mathrm{C}$. Cells exposed to PBS only were included as untreated controls. After removal of the unbound liposomes or free DTX, the cells were incubated with fresh medium at $37^{\circ} \mathrm{C}$ for an additional 46 hours. To determine cell viability, $20 \mu \mathrm{L}$ of MTT solution ( $5 \mathrm{mg} / \mathrm{mL}$ in PBS) was added to each well. Purple formazan was generated after incubation at $37^{\circ} \mathrm{C}$ for 4 hours and was dissolved in $100 \mu \mathrm{L}$ of DMSO, followed by absorbance measurement at $570 \mathrm{~nm}$ using the Benchmark Plus Microplate Reader (Bio-rad Laboratories). Cell viability was expressed as a percentage relative to the absorbance value from untreated cells.

\section{Data analyses}

Data were expressed as mean \pm standard deviation. All statistical analyses were performed with Sigmastat software (Version 3.5, Systat Software, San Jose, CA, USA). The differences among three groups were evaluated using oneway analysis of variance followed by the Student-NewmanKeuls test for post hoc comparisons. A $P$-value below 0.05 indicated that the differences were statistically significant.

\section{Results \\ Coupling of anti-CA IX antibody to liposomes}

CA IX-directed immunoliposomes were prepared by incorporation of antibody-conjugated micelles into DTX-loaded liposomes. Unincorporated micelles or free antibody were removed from the immunoliposomes by Sepharose CL-4B gel filtration chromatography. A total of 15 fractions $(1 \mathrm{~mL}$ per fraction) were eluted from the gel column and were evaluated by UPLC and SDS-PAGE analyses. The immunoliposomes were eluted in Fraction 4-6 and were identified by the turbid appearance of liposome suspension and the presence of encapsulated DTX detected by UPLC (Figure 1A). Free antibody was detected in Fraction 7-11 using SDS-PAGE, whereas lipid-conjugated antibody was detected in Fraction 4-6 (Figure 1B), indicating that antibody-conjugated micelles were indeed incorporated into the liposomes. To estimate the liposome-antibody coupling efficiency, the immunoliposome samples with and without gel filtration were compared using SDS-PAGE (Figure 1C). Serial dilutions of the unfiltered immunoliposome sample with 2, 1, 0.5, and $0.25 \mu \mathrm{g}$ of anti-CA IX antibody were used as calibration standards (Lane 2-5). The eluted immunoliposome fraction (Lane 6), with the absence of unincorporated antibodyconjugated micelles or free antibody, was analyzed at the same amount of DTX as the unfiltered immunoliposome 


\section{A}

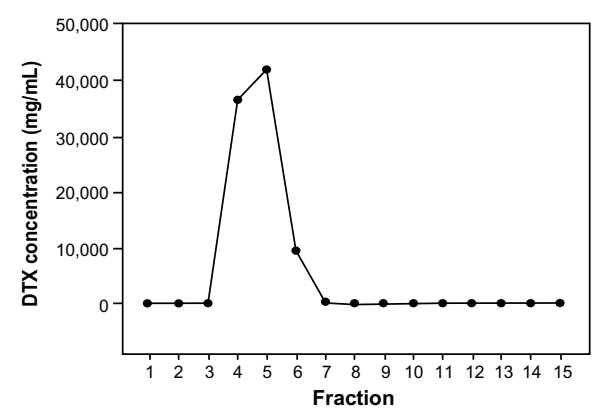

B

Fraction $1 \begin{array}{lllllllllllllll}2 & 2 & 4 & 5 & 6 & 7 & 8 & 9 & 10 & 11 & 12 & 13 & 14 & 15\end{array}$

C

Anti-CA IX Ab

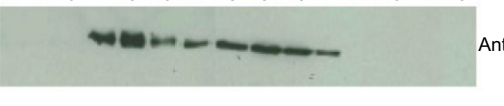

Lane $\begin{array}{llllll}1 & 2 & 3 & 4 & 5 & 6\end{array}$

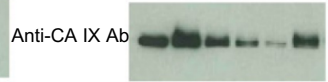

Figure I Separation of CA IX-directed immunoliposomes from free antibody and unincorporated micelles by Sepharose CL-4B gel filtration.

Notes: (A) DTX analysis of the eluted fractions using UPLC. Immunoliposomes encapsulating DTX were detected in Fraction 4-6. (B) SDS-PAGE analysis of anti-CA IX antibody in the eluted fractions. Fraction 4-6 indicated that the anti-CA IX antibody was successfully coupled to the surface of liposomes, whereas Fraction 7-II represented the free antibody that had been removed from the immunoliposomes. (C) Determination of the liposome-antibody coupling efficiency by SDS-PAGE analysis. The eluted immunoliposome fraction (Lane 6) was compared to the calibration standards generated by serial dilution of the unfiltered immunoliposome sample containing $2 \mu \mathrm{g}$ (Lane 2 ), I $\mu \mathrm{g}$ (Lane 3), $0.5 \mu \mathrm{g}$ (Lane 4), and $0.25 \mu \mathrm{g}$ (Lane 5) of antibody. An aliquot of antibody stock was used as a positive control (Lane I). With the same amount of DTX as the $2 \mu \mathrm{g}$ standard (Lane 2), the eluted immunoliposome fraction (Lane 6) was found to contain antibody comparable to the I $\mu \mathrm{g}$ standard (Lane 3), indicating that the coupling efficiency was nearly $50 \%$.

Abbreviations: Ab, antibody; CA IX, carbonic anhydrase IX; SDS-PAGE, sodium dodecyl sulfate polyacrylamide gel electrophoresis; UPLC, ultra-performance liquid chromatography; DTX, docetaxel.

sample containing $2 \mu \mathrm{g}$ of antibody (Lane 2). The amount of antibody observed for the eluted immunoliposome sample (Lane 6) was comparable to the unfiltered $1 \mu \mathrm{g}$ standard (Lane 3), suggesting that the liposome-antibody coupling efficiency was nearly $50 \%$.

\section{Characterization of CA IX-directed immunoliposomes}

Before conjugation with the anti-CA IX antibody, the average particle size of DSPE-PEG-MAL micelles was $17.2 \pm 3.3 \mathrm{~nm}$. The antibody-conjugated micelles were incorporated into DTX liposomes that were sized at $135.3 \pm 8.9 \mathrm{~nm}$ in average diameter. As shown in Table 1, after incorporation of the antibody-conjugated micelles, there was a small but significant increase in the particle size of the DTX liposomes, from $135.3 \pm 8.9 \mathrm{~nm}$ to $143.9 \pm 11.1 \mathrm{~nm}$

Table I Characteristics of DTX liposomes before and after antibody coupling

\begin{tabular}{|c|c|c|c|c|}
\hline & \multicolumn{2}{|c|}{$\begin{array}{l}\text { Particle size } \\
(\mathrm{nm})\end{array}$} & \multicolumn{2}{|c|}{$\begin{array}{l}\text { Encapsulation } \\
\text { efficiency (\%) }\end{array}$} \\
\hline & Mean & SD & Mean & SD \\
\hline DTX liposomes & 135.3 & 8.9 & 84.4 & 3.9 \\
\hline $\begin{array}{l}\text { DTX liposomes with } \\
\text { incorporated micelles } \\
\text { (nontargeted liposomes) }\end{array}$ & 139.0 & 9.7 & - & - \\
\hline $\begin{array}{l}\text { DTX liposomes with } \\
\text { incorporated antibody- } \\
\text { micelles (targeted liposomes) }\end{array}$ & $143.9^{\mathrm{a}}$ & 11.1 & - & - \\
\hline
\end{tabular}

Notes: a $P<0.05$ when compared with the DTX liposome group using one-way repeated measures ANOVA with Student-Newman-Keuls test.

Abbreviations: ANOVA, analysis of variance; DTX, docetaxel; SD, standard deviation.
$(P<0.05)$, suggesting the presence of antibody molecules on the liposome surface. On the other hand, no significant difference in particle size was observed between unmodified liposomes $(135.3 \pm 8.9 \mathrm{~nm})$ and liposomes with incorporated micelles only $(139.0 \pm 9.7 \mathrm{~nm})$. The drug EE was determined to be $84.4 \pm 3.9 \%$. Drug leakage resulting from the postinsertion of antibody-conjugated micelles was considered to be negligible because after Sepharose CL-4B gel filtration, the amount of DTX observed in the eluted immunoliposome fractions (Fraction 4-6, Figure 1A) corresponded to the initial amount of DTX in the unfiltered immunoliposome sample that had been applied to the gel column (96.4\%).

\section{Cell binding of CA IX-directed immunoliposomes}

A fluorescence-based cell binding assay was performed to evaluate the targeting potential of the CA IX-directed immunoliposomes. Hypoxic conditions were used to induce the expression of CA IX in A549 cells (Figure 2A). CA IX remained to be detectable even after 2.5 hours of reoxygenation, indicating that once expressed, the antigen protein was relatively stable (Figure $2 \mathrm{~B}$ ). In the cell binding assay, CA IX-positive cells (after hypoxic treatment) and CA IX-negative cells (after normoxic treatment) were incubated with DiR-labeled liposomes with and without coupling of the anti-CA IX antibody at $4^{\circ} \mathrm{C}$ for 2 hours. After the removal of unbound liposomes, fluorescence intensities were measured using a flow cytometer. Cells treated with PBS only were used as controls to quantify the background signals. As shown 
A

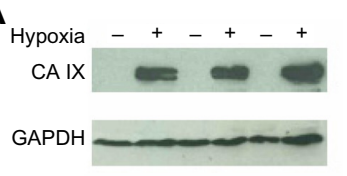

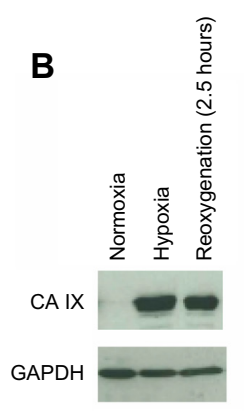

C

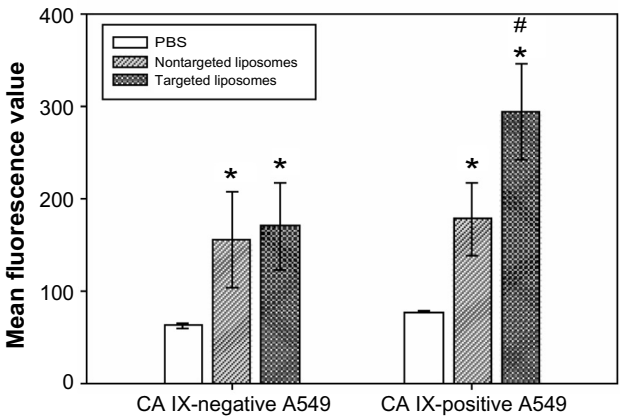

Figure 2 In vitro binding of CA IX-directed immunoliposomes (targeted liposomes) and nontargeted liposomes to human non-small-cell lung cancer cells A549. Notes: (A) Induction of CA IX in triplicate A549 cell cultures by incubation under hypoxic conditions for 20 hours. (B) Detection of CA IX in A549 cells after hypoxic treatment for 20 hours and subsequent reoxygenation for 2.5 hours. CA IX protein was detected by Western blot analysis, with GAPDH as the loading control. (C) Fluorescencebased flow cytometry analysis of the binding activities of the targeted and nontargeted liposomes in CA IX-positive and CA IX-negative A549 cells. Parallel incubations with DiR-labeled liposomes (targeted or nontargeted) and PBS (control) were carried out at $4^{\circ} \mathrm{C}$ for 2 hours. Each treatment was performed in triplicate cultures. The cells were then washed with PBS and collected for measurement of fluorescence intensities by flow cytometry. Data were expressed as mean \pm SD. $* P<0.05$ when compared with the control group and ${ }^{\# P}<0.05$ when compared with the nontargeted liposome group using one-way ANOVA with Student-Newman-Keuls test.

Abbreviations: ANOVA, analysis of variance; CA IX, carbonic anhydrase IX; DiR, I,I'-dioctadecyltetramethyl indotricarbocyanine iodide; GAPDH, glyceraldehyde 3-phosphate dehydrogenase; PBS, phosphate buffered saline; SD, standard deviation.

in Figure 2C, significantly higher fluorescence intensities were observed in cells treated with targeted or nontargeted liposomes when compared with the control group $(P<0.05)$, signifying the successful uptake of the DiR-labeled liposomes in A549 cells. In the absence of CA IX antigen, both targeted and nontargeted liposomes showed similar binding affinities to A549 cells $(P=0.645)$. In contrast, when CA IX was induced, the targeted liposomes were found to exhibit 1.65-fold higher binding affinity than the nontargeted liposomes $(P=0.01)$. These data indicated that antibody coupling to liposomal surface could enhance liposomal uptake in lung cancer cells by immunobinding to CA IX on the cell surface.

\section{Cytotoxic activity of CA IX-directed immunoliposomes}

The anticancer effect of CA IX-directed immunoliposomal DTX was compared with those of nontargeted liposomal DTX and free DTX in A549 cells with and without the expression of CA IX. No significant difference was observed among the three treatment groups at various concentrations in the CA IX-negative cells (Figure 3A), indicating that the anticancer efficacy of liposome-mediated delivery was equivalent to that of free DTX solution when cells were under normoxic conditions. After CA IX was induced in response to hypoxia, nontargeted liposomal DTX appeared
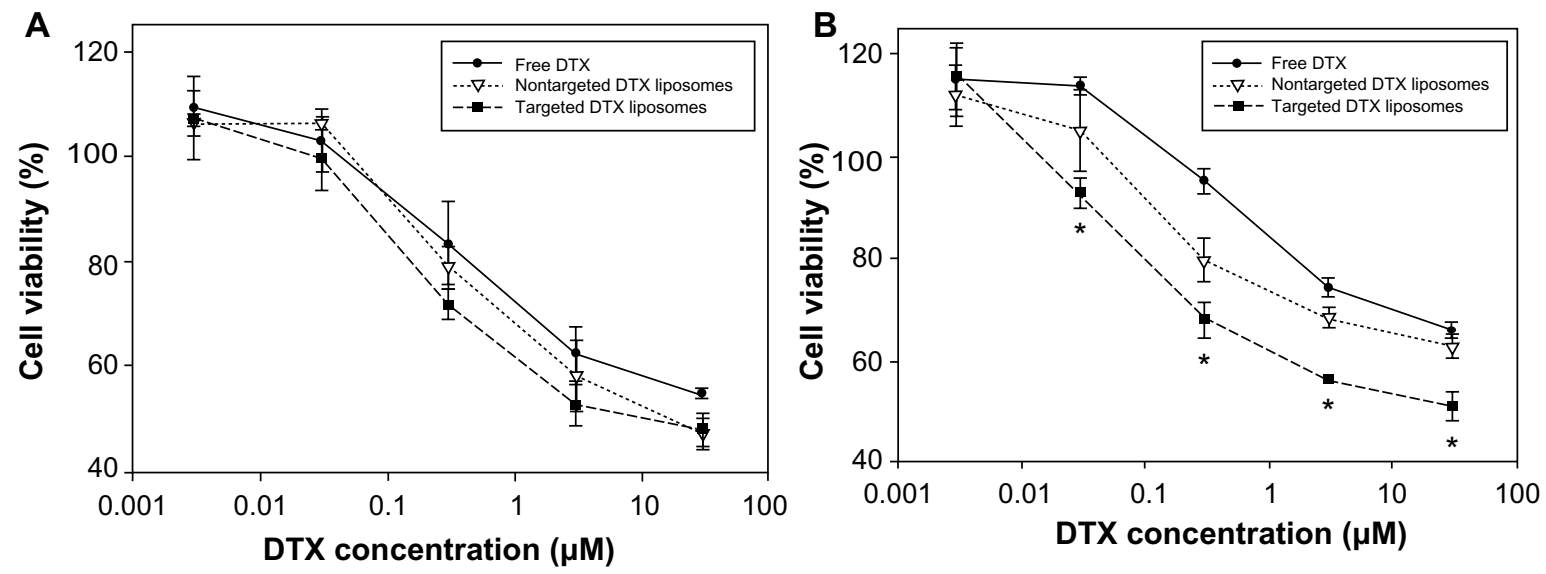

Figure 3 In vitro cytotoxicities of CA IX-directed immunoliposomes (targeted liposomes) and nontargeted liposomes containing DTX in CA IX-negative A549 cells (A) and CA IX-positive A549 cells (B).

Notes: CA IX expression was induced by incubation under hypoxic conditions for 20 hours. Parallel incubations with liposomal DTX (targeted or nontargeted) and free DTX solution were carried out at $4^{\circ} \mathrm{C}$ for 2 hours. Each treatment was performed in triplicate cultures. The cells were then washed with PBS and incubated with fresh medium at $37^{\circ} \mathrm{C}$ for an additional 46 hours. Cell viability was measured by the MTT assay and expressed as a percentage relative to the absorbance of the untreated control. Data were presented as mean \pm SD. $* P<0.05$ when compared with the nontargeted liposome group or free DTX group using one-way ANOVA with Student-Newman-Keuls test. Abbreviations: ANOVA, analysis of variance; CA IX, carbonic anhydrase IX; DTX, docetaxel; MTT, 3-(4,5-dimethylthiazol-2-yl)-2,5-diphenyltetrazolium bromide; PBS, phosphate buffered saline; SD, standard deviation. 
to be more cytotoxic than free DTX, and in comparison with nontargeted treatment, targeted liposomal DTX was found to exhibit an even stronger inhibitory effect on cell growth at different concentrations $(0.03-30 \mu \mathrm{M})$ (Figure 3B, $P<0.05)$. The inhibitory rates for targeted liposomal DTX were higher in a range of $12.1 \%-27.1 \%$ and $8.9 \%-12.2 \%$ than those for free DTX and nontargeted liposomal DTX, respectively. Blank liposomes were diluted to the same SPC concentrations as the DTX-encapsulated liposomes described above and tested on both normoxic and hypoxic A549 cells; no significant toxicities were observed under the same conditions (data not shown). These results suggested that CA IX-mediated liposomal uptake in lung cancer cells could increase drug internalization and thus contribute to a higher anticancer activity.

\section{Discussion}

Targeted liposomal drug delivery has emerged as a promising method for cancer therapy in recent years. ${ }^{27}$ One feasible approach for conferring targeting capability to liposomes is by conjugation of antibody molecules to form immunoliposomes. CA IX is a cell surface protein that is present in many types of solid tumors. ${ }^{2-5} \mathrm{CA}$ IX as a therapeutic antigen target for immunoliposomes has been explored previously for the treatment of renal cell carcinoma; ${ }^{21}$ however, further investigation on CA IX as a feasible target for other types of cancer is lacking. The present study is the first to demonstrate that immunoliposomes directing against CA IX could enhance drug delivery to lung cancer cells.

In this study, CA IX-directed DTX-entrapped immunoliposomes were shown to have a higher cell binding affinity and a stronger antiproliferative effect than the nontargeted liposomes in CA IX-positive lung cancer cells, whereas parallel experiments with CA IX-negative lung cancer cells demonstrated no such differences between the two groups. Expression of CA IX in non-renal-cell carcinomas is known to be induced by hypoxia and is functionally implicated in $\mathrm{pH}$ regulation and cell adhesion supporting the adaptation of tumor cells to hypoxic stress. ${ }^{1}$ Our data suggested that the targeting capability conferred by the anti-CA IX antibody on the liposomal surface would increase drug uptake in hypoxic tumor cells where CA IX is expressed.

Targeting of anticancer drugs to hypoxic tumor cells presents a challenge, given that these cells are spatially distant from blood vessels; however, use of immunoliposomes as a drug delivery system may overcome the problem of poorly perfused hypoxic tissue. Efficient penetration of liposomal drugs to hypoxic regions in the tumor can rely on passive targeting via the enhanced permeability and retention (EPR) effect of liposomes at the leaky vasculature. ${ }^{28}$ To avoid clearance by the renal system and to facilitate efficient extravasation through the leaky vasculature, liposomes should theoretically be in the range of $10-400 \mathrm{~nm}$ in size. ${ }^{29,30} \mathrm{In}$ this study, the average particle size of the CA IX-directed immunoliposomes was measured to be $143.9 \pm 11.1 \mathrm{~nm}$, large enough to avoid elimination in the kidney and small enough to extravasate through capillary fenestrations into the tumor interstitium. In addition, the EPR effect could be enhanced with the presence of PEG on the surface of liposomes (through the use of DSPE-PEG-MAL in the formulation), which would reduce macrophage uptake and prolong bioavailability. ${ }^{31}$ Subsequent to the EPR effect, active targeting via interaction with cell surface CA IX antigen would increase immunoliposomal drug localization within the hypoxic regions in the tumor.

Binding of immunoliposomes to tumor cells is followed by cytoplasmic drug accumulation, which exerts the cytotoxic activity. Endocytosis has been implicated as the mode of cell entry for liposomes. ${ }^{32}$ Uptake of immunoliposomal drug via endocytosis is facilitated by antigen binding on the cell surface, resulting in higher intracellular drug accumulation and thus increased cytotoxicity in comparison to treatment with nontargeted liposomal drug. In addition, use of immunoliposomes for targeting hypoxic cells may help resolve the problem of multidrug resistance in cancer. The expression of the transporter protein P-glycoprotein (P-gp) has been shown to be correlated with hypoxia in vitro and in vivo, promoting multidrug resistance in cancer cells. ${ }^{33-36}$ In multidrug resistant cells, formation of endosomes could direct liposomal drug away from the membrane-bound P-gp efflux pump and localize drug release near the perinuclear region, allowing efficient trafficking into the nucleus. ${ }^{37}$ Free drugs, on the other hand, are internalized by diffusion across the cell membrane, making them spatially more susceptible to capture and efflux by P-gp. ${ }^{38}$ As shown in the present study (Figure 3), similar cytotoxicities were observed for free DTX and liposomal DTX (targeted or nontargeted) in normoxia-treated cancer cells; on the other hand, hypoxiatreated cancer cells appeared to be more resistant to free DTX in comparison to liposomal DTX. This observation could be attributed to the increased expression of P-gp under hypoxic conditions, causing relatively more efflux for free drug molecules. ${ }^{36}$ With the ability to accumulate inside the cells more readily through immunobinding to cell surface CA IX, targeted liposomal DTX was found to exhibit even higher cytoxicities than nontargeted liposomal DTX at a wide 
range of concentrations in CA IX-expressed cancer cells. These data suggested that drug delivery to hypoxic cells via CA IX-directed immunoliposomes could be a promising strategy for reversal of multidrug resistance in cancer.

Future work would explore the potential use of CA IXdirected immunoliposomes for drug delivery in various tumor-bearing mouse models. As CA IX is found to be expressed in different types of solid tumors, the development of CA IX-directed immunoliposomes would have a wide scope of application in targeted cancer therapy. Particularly, lung cancer is known to be the leading cause of cancerrelated deaths worldwide. ${ }^{39}$ Finding an effective treatment for lung cancer has thus been an important goal in clinical medicine. Further investigation may be performed to evaluate the therapeutic efficacies of different anticancer agents using CA IX-directed immunoliposomes as a carrier system in an orthotopic lung cancer model. ${ }^{40}$ Immunoliposomal drugs may be administered via the intratracheal route for direct delivery to lung tumors ${ }^{41}$ and minimizing the potential interference by soluble CA IX present in the circulation. ${ }^{26}$ Our preliminary in vivo study based on another anticancer drug formulated in CA IX-directed immunoliposomes has shown promising results for the treatment of lung cancer (data not shown). In addition, the amount of antibody molecules bound to the liposomal surface can be further optimized to enhance drug uptake by CA IX-expressed cancer cells. Previous reports have demonstrated that an increase in the liposomal antibody density by adjusting the molar ratio of linker lipid to antibody in the conjugation reaction and the molar percentage of linker lipid incorporated into liposomes could raise the binding efficiencies of immunoliposomes towards cancer cells in vitro. ${ }^{42,43}$ Therefore, in vivo studies along with comparison of formulations based on various liposomal antibody densities are anticipated to further determine whether CA IX-directed immunoliposomes can increase drug accumulation in the tumor, leading to a higher therapeutic response.

\section{Conclusion}

This study is the first to demonstrate that CA IX-directed immunoliposomes can be a useful drug delivery tool for targeting lung cancer cells. Monoclonal antibodies specific to human CA IX antigen were successfully coupled to the surface of drug-loaded liposomes to form immunoliposomes. In vitro studies showed that antibody conjugation of liposomes could significantly increase their uptake by CA IX-positive cancer cells and enhance the antiproliferative effect of the encapsulated drug. These results have important implications for CA IX-mediated drug delivery as a potential strategy to improve the efficacy of current anticancer therapies. Furthermore, the present work can serve as a model for similar studies to be carried out on other types of CA IX-associated tumors, opening up new possibilities in the application of immunoliposomes for targeted cancer therapy.

\section{Acknowledgments}

We would like to thank Hong Kong Baptist University for the Faculty Research Grant support. We would also like to express our gratitude to the Department of Anatomical and Cellular Pathology, The Chinese University of Hong Kong, for providing us the human lung cancer cell line.

\section{Disclosure}

The authors report no conflicts of interest in this work.

\section{References}

1. Pastorekova S, Zatovicova M, Pastorek J. Cancer-associated carbonic anhydrases and their inhibition. Curr Pharm Des. 2008;14(7): 685-698.

2. Liao SY, Aurelio ON, Jan K, Zavada J, Stanbridge EJ. Identification of the MN/CA9 protein as a reliable diagnostic biomarker of clear cell carcinoma of the kidney. Cancer Res. 1997;57(14):2827-2831.

3. Saarnio J, Parkkila S, Parkkila AK, et al. Immunohistochemical study of colorectal tumors for expression of a novel transmembrane carbonic anhydrase, MN/CA IX, with potential value as a marker of cell proliferation. Am J Pathol. 1998;153(1):279-285.

4. Wykoff CC, Beasley N, Watson PH, et al. Expression of the hypoxia-inducible and tumor-associated carbonic anhydrases in ductal carcinoma in situ of the breast. Am J Pathol. 2001;158(3): 1011-1019.

5. Vermylen P, Roufosse C, Burny A, et al. Carbonic anhydrase IX antigen differentiates between preneoplastic malignant lesions in non-small cell lung carcinoma. Eur Respir J. 1999;14(4):806-811.

6. Loncaster JA, Harris AL, Davidson SE, et al. Carbonic anhydrase (CA IX) expression, a potential new intrinsic marker of hypoxia: correlations with tumor oxygen measurements and prognosis in locally advanced carcinoma of the cervix. Cancer Res. 2001;61(17):6394-6399.

7. Ilie M, Mazure NM, Hofman V, et al. High levels of carbonic anhydrase IX in tumour tissue and plasma are biomarkers of poor prognostic in patients with non-small cell lung cancer. Br J Cancer. 2010;102(11):1627-1635.

8. Chia SK, Wykoff CC, Watson PH, et al. Prognostic significance of a novel hypoxia-regulated marker, carbonic anhydrase IX, in invasive breast carcinoma. J Clin Oncol. 2001;19(16):3660-3668.

9. Måseide K, Kandel RA, Bell RS, et al. Carbonic anhydrase IX as a marker for poor prognosis in soft tissue sarcoma. Clin Cancer Res. 2004;10(13):4464-4471.

10. Haapasalo JA, Nordfors KM, Hilvo M, et al. Expression of carbonic anhydrase IX in astrocytic tumors predicts poor prognosis. Clin Cancer Res. 2006;12(2):473-477.

11. Le QT, Chen E, Salim A, et al. An evaluation of tumor oxygenation and gene expression in patients with early stage non-small cell lung cancers. Clin Cancer Res. 2006;12(5):1507-1514.

12. Swinson DE, Jones JL, Richardson D, et al. Carbonic anhydrase IX expression, a novel surrogate marker of tumor hypoxia, is associated with a poor prognosis in non-small-cell lung cancer. J Clin Oncol. 2003;21(3):473-482. 
13. Pastoreková S, Parkkila S, Parkkila AK, et al. Carbonic anhydrase IX, MN/CA IX: analysis of stomach complementary DNA sequence and expression in human and rat alimentary tracts. Gastroenterology. 1997;112(2):398-408.

14. Kivelä AJ, Parkkila S, Saarnio J, et al. Expression of transmembrane carbonic anhydrase isoenzymes IX and XII in normal human pancreas and pancreatic tumours. Histochem Cell Biol. 2000;114(3):197-204.

15. Karhumaa P, Kaunisto K, Parkkila S, et al. Expression of the transmembrane carbonic anhydrases, CA IX and CA XII, in the human male excurrent ducts. Mol Hum Reprod. 2001;7(7):611-616.

16. Surfus JE, Hank JA, Oosterwijk E, et al. Anti-renal-cell carcinoma chimeric antibody G250 facilitates antibody-dependent cellular cytotoxicity with in vitro and in vivo interleukin-2-activated effectors. J Immunother Emphasis Tumor Immunol. 1996;19(3):184-191.

17. Siebels M, Rohrmann K, Oberneder R, et al. A clinical phase I/II trial with the monoclonal antibody cG250 (RENCAREX $\left.{ }^{\circledR}\right)$ and interferonalpha-2a in metastatic renal cell carcinoma patients. World J Urol. 2011;29(1):121-126.

18. Zatovicova M, Jelenska L, Hulikova A, et al. Carbonic anhydrase IX as an anticancer therapy target: preclinical evaluation of internalizing monoclonal antibody directed to catalytic domain. Curr Pharm Des. 2010;16(29):3255-3263.

19. Torchilin VP. Recent advances with liposomes as pharmaceutical carriers. Nat Rev Drug Discov. 2005;4(2):145-160.

20. Kontermann RE. Immunoliposomes for cancer therapy. Curr Opin Mol Ther. 2006;8(1):39-45.

21. Shinkai M, Le B, Honda H, et al. Targeting hyperthermia for renal cell carcinoma using human $\mathrm{MN}$ antigen-specific magnetoliposomes. Jpn J Cancer Res. 2001;92(10):1138-1145.

22. Wykoff CC, Beasley NJ, Watson PH, et al. Hypoxia-inducible expression of tumor-associated carbonic anhydrases. Cancer Res. 2000;60(24):7075-7083.

23. Pons M, Foradada M, Estelrich J. Liposomes obtained by the ethanol injection method. Int J Pharm. 1993;95(1-3):51-56.

24. Iden DL, Allen TM. In vitro and in vivo comparison of immunoliposomes made by conventional coupling techniques with those made by a new post-insertion approach. Biochim Biophys Acta. 2001; 1513(2):207-216.

25. Manjappa AS, Chaudhari KR, Venkataraju MP, et al. Antibody derivatization and conjugation strategies: application in preparation of stealth immunoliposome to target chemotherapeutics to tumor. $J$ Control Release. 2011;150(1):2-22.

26. Závada J, Závadová Z, Zat'ovicová M, Hyrsl L, Kawaciuk I. Soluble form of carbonic anhydrase IX (CA IX) in the serum and urine of renal carcinoma patients. Br J Cancer. 2003;89(6):1067-1071.

27. Medina OP, Zhu Y, Kairemo K. Targeted liposomal drug delivery in cancer. Curr Pharm Des. 2004;10(24):2981-2989.

28. Iyer AK, Khaled G, Fang J, Maeda H. Exploiting the enhanced permeability and retention effect for tumor targeting. Drug Discov Today. 2006;11(17-18):812-818.
29. Danhier F, Feron O, Préat V. To exploit the tumor microenvironment: Passive and active tumor targeting of nanocarriers for anti-cancer drug delivery. J Control Release. 2010;148(2):135-146.

30. Choi HS, Liu W, Misra P, et al. Renal clearance of quantum dots. Nat Biotechnol. 2007;25(10):1165-1170.

31. Immordino ML, Dosio F, Cattel L. Stealth liposomes: review of the basic science, rationale, and clinical applications, existing and potential. Int J Nanomedicine. 2006;1(3):297-315.

32. Sheehan JP, Sheehan JM, Holmberg EG, Geisert EE, Helm GA. A horseradish peroxidase-light and electron microscopic study of immunoliposomes utilized for intracellular delivery to the rat striatum. Neurosci Lett. 2002;333(3):212-216.

33. Mellor HR, Callaghan R. Accumulation and distribution of doxorubicin in tumour spheroids: the influence of acidity and expression of P-glycoprotein. Cancer Chemother Pharmacol. 2011;68(5): 1179-1190.

34. Thews O, Nowak M, Sauvant C, Gekle M. Hypoxia-induced extracellular acidosis increases p-glycoprotein activity and chemoresistance in tumors in vivo via p38 signaling pathway. Adv Exp Med Biol. 2011;701: $115-122$.

35. Xiao-Dong L, Zhi-Hong Y, Hui-Wen Y. Repetitive/temporal hypoxia increased P-glycoprotein expression in cultured rat brain microvascular endothelial cells in vitro. Neurosci Lett. 2008;432(3):184-187.

36. Xia S, Yu S, Yuan X. Effects of hypoxia on expression of P-gp and mutltidrug resistance protein in human lung adenocarcinoma A549 cell line. J Huazhong Univ Sci Technolog Med Sci. 2005;25(3): 279-281.

37. Omelyanenko V, Kopecková P, Gentry C, Kopecek J. Targetable HPMA copolymer-adriamycin conjugates. Recognition, internalization, and subcellular fate. J Control Release. 1998;53(1-3):25-37.

38. Shen F, Chu S, Bence AK, et al. Quantitation of doxorubicin uptake, efflux, and modulation of multidrug resistance (MDR) in MDR human cancer cells. J Pharmacol Exp Ther. 2008;324(1):95-102.

39. Baas P, Belderbos JS, van den Heuvel M. Chemoradiation therapy in nonsmall cell lung cancer. Curr Opin Oncol. 2011;23(2):140-149.

40. Onn A, Isobe T, Itasaka S, et al. Development of an orthotopic model to study the biology and therapy of primary human lung cancer in nude mice. Clin Cancer Res. 2003;9(15):5532-5539.

41. Gagnadoux F, Pape AL, Lemarié E, et al. Aerosol delivery of chemotherapy in an orthotopic model of lung cancer. Eur Respir J. 2005;26(4):657-661.

42. Mercadal M, Domingo JC, Petriz J, Garcia J, de Madariaga MA. Preparation of immunoliposomes bearing poly(ethylene glycol)-coupled monoclonal antibody linked via a cleavable disulfide bond for ex vivo applications. Biochim Biophys Acta. 2000;1509(1-2):299-310.

43. Mercadal M, Domingo JC, Petriz J, Garcia J, de Madariaga MA. A novel strategy affords high-yield coupling of antibody to extremities of liposomal surface-grafted PEG chains. Biochim Biophys Acta. 1999;1418(1):232-238.
Drug Design, Development and Therapy

\section{Publish your work in this journal}

Drug Design, Development and Therapy is an international, peerreviewed open-access journal that spans the spectrum of drug design and development through to clinical applications. Clinical outcomes, patient safety, and programs for the development and effective, safe, and sustained use of medicines are a feature of the journal, which

\section{Dovepress}

has also been accepted for indexing on PubMed Central. The manuscript management system is completely online and includes a very quick and fair peer-review system, which is all easy to use. Visit http://www.dovepress.com/testimonials.php to read real quotes from published authors. 